\title{
On homoclinic orbits for a class of damped vibration systems
}

\author{
Juntao Sun ${ }^{1 *}$, Juan J Nieto ${ }^{2,3}$ and Mario Otero-Novoa ${ }^{2}$
}

\section{"Correspondence:}

sunjuntao2008@163.com

'School of Science, Shandong

University of Technology, Zibo,

Shandong 255049, China

Full list of author information is

available at the end of the article

\begin{abstract}
In this article, we establish the new result on homoclinic orbits for a class of damped vibration systems. Some recent results in the literature are generalized and significantly improved.

MSC: Primary 49J40; secondary $70 \mathrm{H} 05$

Keywords: homoclinic orbits; second-order systems; damped vibration problems; variational methods; $(C)_{c}$-sequence
\end{abstract}

\section{Introduction and main results}

Consider the following second-order damped vibration problems

$$
\ddot{u}(t)+B \dot{u}(t)-L(t) u(t)+W_{u}(t, u(t))=0, \quad t \in \mathbb{R},
$$

where $u=\left(u_{1}, u_{2}, \ldots, u_{N}\right) \in \mathbb{R}^{N}, B$ is an antisymmetric $N \times N$ constant matrix, $L \in$ $C\left(\mathbb{R}, \mathbb{R}^{N \times N}\right)$ is a symmetric matrix valued function and $W \in C^{1}\left(\mathbb{R} \times \mathbb{R}^{N}, \mathbb{R}\right)$. As usual we say that a solution $u$ of (VS) is homoclinic (to 0 ) if $u \in C^{2}\left(\mathbb{R}, \mathbb{R}^{N}\right), u \neq 0, u(t) \rightarrow 0$, and $\dot{u}(t) \rightarrow 0$ as $|t| \rightarrow \infty$.

When $B$ is a zero matrix, (VS) is just the following second-order Hamiltonian systems (HSs)

$$
\ddot{u}(t)-L(t) u(t)+W_{u}(t, u(t))=0, \quad t \in \mathbb{R} .
$$

Inspired by the excellent monographs and works [1-3], by now, the existence and multiplicity of periodic and homoclinic solutions for HSs have extensively been investigated in many articles via variational methods, see [4-22]. Also second-order HSs with impulses via variational methods have recently been considered in [23-26]. More precisely, in 1990, Rabinowitz [3] established the existence result on homoclinic orbit for the periodic second-order HS. It is well known that the periodicity is used to control the lack of compactness due to the fact that HS is set on all $\mathbb{R}$.

For the nonperiodic case, the problem is quite different from the one described in nature. Rabinowitz and Tanaka [13] introduced a type of coercive condition on the matrix $L$ :

$\left(L_{1}\right) l(t):=\inf _{|x|=1} L(t) x \cdot x \rightarrow+\infty$, as $|t| \rightarrow \infty$. 
They established a compactness lemma under the nonperiodic case and obtained the existence of homoclinic orbit for the nonperiodic system (HS) under the usual AmbrosettiRabinowitz (AR) growth condition

$$
0<\mu W(t, u) \leq W_{u}(t, u(t)) u, \quad \forall t \in \mathbb{R} \text { and } u \in \mathbb{R}^{N} \backslash\{0\},
$$

where $\mu>2$ is a constant. Later, Ding [7] strengthened condition $\left(L_{1}\right)$ by

$\left(L_{2}\right)$ there exists a constant $\alpha>0$ such that

$$
l(t)|t|^{-\alpha} \rightarrow+\infty \quad \text { as }|t| \rightarrow \infty
$$

Under the condition $\left(L_{2}\right)$ and some subquadratic conditions on $W(t, u)$, Ding proved the existence and multiplicity of homoclinic orbits for the system (HS). From then on, the condition $\left(L_{1}\right)$ or $\left(L_{2}\right)$ are extensively used in many articles.

Compared with the case where $B$ is a zero matrix, the case where $B \neq 0$, i.e., the nonperiodic system (VS), has been considered only by a few authors, see [27-29]. Zhang and Yuan [28] studied the existence of homoclinic orbits for the nonperiodic system (VS) when $W$ satisfies the subquadratic condition at infinity. Soon after, Wu and Zhang [27] obtained the existence and multiplicity of homoclinic orbits for the nonperiodic system (VS) when $W$ satisfies the local (AR) growth condition

$$
0<\mu W(t, u) \leq W_{u}(t, u) u, \quad \forall t \in \mathbb{R} \text { and }|u| \geq r
$$

where $\mu>2$ and $r>0$ are two constants. It is worth noticing that the matrix $L$ is required to satisfy the condition $\left(L_{1}\right)$ in the above two articles.

Inspired by $[27,28]$, in this article we shall replace the condition $\left(L_{1}\right)$ on $L$ by the following conditions:

$\left(L_{3}\right)$ there exists a constant $\beta>1$ such that

$$
\text { meas }\left\{t \in \mathbb{R}:|t|^{-\beta} L(t)<b I_{N}\right\}<+\infty, \quad \forall b>0,
$$

and

$\left(L_{4}\right)$ there exists a constant $\gamma \geq 0$ such that

$$
l(t):=\inf _{|x|=1} L(t) x \cdot x \geq-\gamma, \quad \forall t \in \mathbb{R}
$$

which are first used in [20]. By using a recent critical point theorem, we prove that the nonperiodic system (VS) has at least one homoclinic orbit when $W$ satisfies weak superquadratic at the infinity, which improve and extend the results of $[27,28]$.

Remark 1 In fact, there are some matrix-valued functions $L(t)$ satisfying $\left(L_{3}\right)$ and $\left(L_{4}\right)$, but not satisfying $\left(L_{1}\right)$ or $\left(L_{2}\right)$. For example,

$$
L(t)=\left(t^{4} \sin ^{2} t+1\right) I_{N}
$$


We consider the following conditions:

$\left(W_{1}\right) \quad W \in C^{1}\left(\mathbb{R} \times \mathbb{R}^{N}, \mathbb{R}\right)$, and there exist positive constants $c_{1}$ and $v>2$ such that

$$
c_{1}|u|^{\nu} \leq W_{u}(t, u) u, \quad \forall(t, u) \in \mathbb{R} \times \mathbb{R}^{N} .
$$

$\left(W_{2}\right) \quad W_{u}(t, u)=o(|u|)$ as $|u| \rightarrow 0$ uniformly in $t$.

$\left(W_{3}\right) \tilde{W}(t, u):=\frac{1}{2} W_{u}(t, u) u-W(t, u)>0$ if $u \neq 0$, and

$$
\inf \left\{\frac{\tilde{W}(t, u)}{|u|^{2}}: t \in \mathbb{R} \text { with } a \leq|u|<b\right\}>0
$$

for any $a, b>0$.

$\left(W_{4}\right)$ There exist $r>0$ and $\sigma>1$ such that $\left|W_{u}(t, u)\right|^{\sigma} \leq c \tilde{W}(t, u)|u|^{\sigma}$ if $|u| \geq r$.

Theorem 2 Assume that $\left(L_{3}\right)-\left(L_{4}\right)$ and $\left(W_{1}\right)-\left(W_{4}\right)$ hold. Then the system $(V S)$ has at least one homoclinic orbit.

Remark 3 To see that our result generalizes [27] we present the following examples. These functions satisfy the weak superquadratic conditions $\left(W_{1}\right)-\left(W_{4}\right)$, but not verify the growth condition (1).

Example:

$$
W(t, u)=a(t)\left(|u|^{p}+(p-2)|u|^{p-\epsilon} \sin ^{2}\left(\frac{|u|^{\epsilon}}{\epsilon}\right)\right),
$$

where $\inf _{t \in \mathbb{R}} a(t)>0$, and $p>2,0<\epsilon<p-2$.

In fact it is easy to verify that $\left(W_{1}\right)-\left(W_{4}\right)$ are satisfied. However, similar to the discussion of Remark 1.2 in [30], let $u_{n}=\left(\epsilon\left(n \pi+\frac{3 \pi}{4}\right)\right)^{\frac{1}{\epsilon}} e_{1}$, where $e_{1}=(1,0, \ldots, 0)$. Then for any $\mu>2$, one has

$$
\begin{aligned}
W_{u}\left(t, u_{n}\right) u_{n}-\mu W\left(t, u_{n}\right)= & a(t)\left[(p-\mu)\left|u_{n}\right|^{p}\right. \\
& +(p-2)(p-\epsilon-\mu)\left|u_{n}\right|^{p-\epsilon} \sin ^{2}\left(\left|u_{n}\right|^{\epsilon} / \epsilon\right) \\
& \left.+(p-2)\left|u_{n}\right|^{p} \sin 2\left(\left|u_{n}\right|^{\epsilon} / \epsilon\right)\right] \\
= & a(t)\left|u_{n}\right|^{p}\left[2-\mu+\frac{(p-2)(p-\epsilon-\mu) \sin ^{2}\left(\left|u_{n}\right|^{\epsilon} / \epsilon\right)}{\left|u_{n}\right|^{\epsilon}}\right] \\
\rightarrow & -\infty \text { as } n \rightarrow \infty .
\end{aligned}
$$

That is, the condition (1) is not satisfied for any $\mu>2$.

This article is organized as follows. In the following section, we formulate the variational setting and recall a critical point theorem required. In section 'Linking structure', we discuss linking structure of the functional. In section 'The $(C)_{c}$-sequence', we study the Cerami condition of the functional and give the proof of Theorem 2.

Notation Throughout the article, we shall denote by $c>0$ various positive constants which may vary from line to line and are not essential to the problem. 


\section{Variational setting}

In this section, we establish a variational setting for the system (VS). Let $H$ be $H^{1}\left(\mathbb{R}, \mathbb{R}^{N}\right)$ which is a Hilbert space with the inner product and norm given by

$$
\langle u, v\rangle_{H}=\int_{\mathbb{R}}[(\dot{u}(t), \dot{v}(t))+(u(t), v(t))] d t
$$

and

$$
\|u\|_{H}=\left(\int_{\mathbb{R}}\left[|\dot{u}(t)|^{2}+|u(t)|^{2}\right] d t\right)^{\frac{1}{2}}
$$

for $u, v \in H$, where $(\cdot, \cdot)$ denotes the inner product in $\mathbb{R}^{N}$. It is well known that $H$ is continuously embedded in $L^{p}\left(\mathbb{R}, \mathbb{R}^{N}\right)$ for $p \in[2, \infty)$. Define an operator $J: H \rightarrow H$ by

$$
\langle u, v\rangle=\int_{\mathbb{R}}(B u, \dot{v}) d t
$$

for all $u, v \in H$. Since $B$ is an antisymmetric $N \times N$ constant matrix, $J$ is self-adjoint on $H$. Moreover, we denote by $A$ the self-adjoint extension of the operator $-\frac{d^{2}}{d t^{2}}+L(t)+J$ with the domain $\mathcal{D}(A) \subset L^{2}\left(\mathbb{R}, \mathbb{R}^{N}\right)$. Let $|\cdot|_{p}$ be the usual $L^{p}$-norm, and $\langle\cdot, \cdot\rangle_{2}$ the usual $L^{2}$-inner product. Set $E:=\mathcal{D}\left(|A|^{\frac{1}{2}}\right)$, the domain of $|A|^{\frac{1}{2}}$. Define on $E$ the inner product

$$
\langle u, v\rangle_{E}:=\left\langle|A|^{\frac{1}{2}} u,|A|^{\frac{1}{2}} v\right\rangle_{2}+\langle u, v\rangle_{2}
$$

and the norm

$$
\|u\|_{E}=\langle u, u\rangle_{E}^{\frac{1}{2}} .
$$

Then $E$ is a Hilbert space and it is easy to verify that $E$ is continuously embedded in $H^{1}\left(\mathbb{R}, \mathbb{R}^{N}\right)$. Using a similar proof of Lemma 3.1 in [20], we can prove the following lemma.

Lemma 4 Suppose that $L(t)$ satisfies $\left(L_{3}\right)$ and $\left(L_{4}\right)$, then $E$ is compactly embedded into $L^{p}\left(\mathbb{R}, \mathbb{R}^{N}\right)$ for $p \in[1,+\infty]$.

By Lemma 4, it is easy to prove that the spectrum $\sigma(A)$ has a sequence of eigenvalues (counted with their multiplicities)

$$
\lambda_{1} \leq \lambda_{2} \leq \cdots \leq \lambda_{k} \leq \cdots
$$

with $\lambda_{k} \rightarrow+\infty$ as $k \rightarrow+\infty$, and corresponding eigenfunctions $\left\{e_{k}\right\}_{k \in \mathbb{N}}, A e_{k}=\lambda_{k} e_{k}$, form an orthogonal basis in $L^{2}\left(\mathbb{R}, \mathbb{R}^{N}\right)$. Assume $\lambda_{1}, \lambda_{2}, \ldots, \lambda_{\ell^{-}}<0, \lambda_{\ell^{-}+1}=\cdots=\lambda_{\ell}=0$ and let $E^{-}:=\operatorname{span}\left\{e_{1}, \ldots, e_{\ell^{-}}\right\}, E^{0}:=\operatorname{span}\left\{e_{\ell^{-}+1}, \ldots, e_{\ell}\right\}$, and $E^{+}:=\operatorname{cl}_{E}\left(\operatorname{span}\left\{e_{\ell+1}, \ldots\right\}\right)$. Then

$$
E=E^{-} \oplus E^{0} \oplus E^{+}
$$

is an orthogonal decomposition of $E$. We introduce on $E$ the following product

$$
\langle u, v\rangle:=\left\langle|A|^{\frac{1}{2}} u,|A|^{\frac{1}{2}} v\right\rangle_{2}+\left\langle u^{0}, v^{0}\right\rangle_{2},
$$


and the norm

$$
\|u\|=\langle u, u\rangle^{\frac{1}{2}}
$$

where $u=u^{-}+u^{0}+u^{+}, v=v^{-}+v^{0}+v^{+} \in E^{-} \oplus E^{0} \oplus E^{+}$. Then $\|\cdot\|$ and $\|\cdot\|_{E}$ are equivalent (see [7]). So by Lemma 4 , we see that there exists a constant $\eta_{p}>0$ such that

$$
|u|_{p} \leq \eta_{p}\|u\|, \quad \forall u \in E, \forall p \in[1,+\infty] .
$$

Define the functional $\Phi$ on $E$ by

$$
\Phi(u)=\int_{\mathbb{R}}\left[\frac{1}{2}|\dot{u}(t)|^{2}+\frac{1}{2}(B u(t), \dot{u}(t))+\frac{1}{2}(L(t) u(t), u(t))-W(t, u(t))\right] d t .
$$

Then

$$
\Phi(u)=\frac{1}{2}\left(\left\|u^{+}\right\|^{2}-\left\|u^{-}\right\|^{2}\right)-\int_{\mathbb{R}} W(t, u(t)) d t,
$$

where $u=u^{-}+u^{0}+u^{+} \in E$. Furthermore, define

$$
\Psi(u):=\int_{\mathbb{R}} W(t, u) d t .
$$

From the assumptions it follows that $\Phi$ is defined on the Banach space $E$ and belongs to $C^{1}(E, \mathbb{R})$. A standard argument shows that critical points of $\Phi$ are solutions of the system (VS). Moreover, it is easy to verify that if $u \neq \equiv 0$ is a solution of (VS), then $u(t) \rightarrow 0$ and $\dot{u}(t) \rightarrow 0$, as $|t| \rightarrow \infty$ (see Lemma 3.1 in [31]).

In order to study the critical points of $\Phi$, we now recall a critical point theorem, see [32].

Let $E$ be a Banach space. A sequence $\left\{u_{n}\right\} \subset E$ is said to be a $(C)_{c}$-sequence if

$$
\Phi\left(u_{n}\right) \rightarrow c \text { and }\left(1+\left\|u_{n}\right\|\right) \Phi^{\prime}\left(u_{n}\right) \rightarrow 0 .
$$

$\Phi$ is said to satisfy the $(C)_{c}$-condition if any $(C)_{c}$-sequence has a convergent subsequence.

Theorem 5 ([32]) Suppose $\Phi \in C^{1}(E, \mathbb{R}), E=X \oplus Y$, where $\operatorname{dim} X\langle\infty$, there exist $R>\rho\rangle$ $0, \kappa>0$ and $e_{0} \in Y \backslash\{0\}$ such that $\inf \Phi\left(Y \cap S_{\rho}\right) \geq \kappa$ and $\sup \Phi(\partial Q) \leq 0$, where $S_{\rho}:=S_{\rho}(0)$ is the sphere of radius $\rho$ and center 0 , and

$$
Q=\left\{u=x+s e_{0}: s \geq 0, x \in X,\|u\| \leq R\right\} .
$$

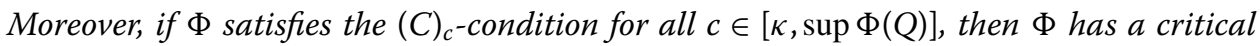
value in $[\kappa, \sup \Phi(Q)]$.

\section{Linking structure}

First we discuss the linking structure of $\Phi$. By condition $\left(W_{1}\right)$, one has

$$
W(t, u) \geq c_{1}|u|^{v} \geq 0
$$


for all $(t, u) \in \mathbb{R} \times \mathbb{R}^{N}$. Observe that if ( $\left.W_{4}\right)$ holds, and together with (4), then if $|u|>r$, one has

$$
\begin{aligned}
\left|W_{u}(t, u)\right|^{\sigma} & \leq c\left(\frac{1}{2} W_{u}(t, u) u-W(t, u)\right)|u|^{\sigma} \\
& \leq \frac{c}{2} W_{u}(t, u) u|u|^{\sigma} \\
& \leq \frac{c}{2}\left|W_{u}(t, u)\right||u|^{\sigma+1},
\end{aligned}
$$

and hence

$$
\left|W_{u}(t, u)\right| \leq\left(\frac{c}{2}\right)^{\frac{1}{\sigma-1}}|u|^{\frac{\sigma+1}{\sigma-1}}, \quad \text { if }|u| \geq r .
$$

Let $p=2 \sigma /(\sigma-1)>2$. Then we have

$$
\left|W_{u}(t, u)\right| \leq\left(\frac{c}{2}\right)^{\frac{1}{\sigma-1}}|u|^{p-1}, \quad \text { if }|u| \geq r .
$$

Remark that $\left(W_{2}\right)$ and (5) imply that, for any $\varepsilon>0$, there is $C_{\varepsilon}>0$ such that

$$
\left|W_{u}(t, u)\right| \leq \varepsilon|u|+C_{\varepsilon}|u|^{p-1},
$$

and

$$
|W(t, u)| \leq \varepsilon|u|^{2}+C_{\varepsilon}|u|^{p}
$$

for all $(t, u) \in \mathbb{R} \times \mathbb{R}^{N}$.

Lemma 6 Let $\left(W_{1}\right)-\left(W_{2}\right)$ be satisfied, and assume further that $\left(W_{4}\right)$ holds. Then there exists $\rho>0$ such that $\kappa:=\inf \Phi\left(S_{\rho}^{+}\right)>0$, where $S_{\rho}^{+}=\partial B_{\rho} \cap E^{+}$.

Proof By (7) we have

$$
\Psi(u) \leq \varepsilon|u|_{2}^{2}+C_{\varepsilon}|u|_{p}^{p} \leq c\left(\varepsilon\|u\|^{2}+C_{\varepsilon}\|u\|^{p}\right)
$$

for all $u \in E$, the lemma follows from the form of $\Phi$ (see (3)).

\section{Denote}

$$
\mathcal{H}:=\mathbb{R} e_{\ell+1}, \quad E_{\mathcal{H}}=E^{-} \oplus E^{0} \oplus \mathcal{H} .
$$

Then $E_{\mathcal{H}}$ is a finite subspace.

Lemma 7 Under the assumptions of Theorem 2, there exists $R_{E_{\mathcal{H}}}>0$ such that $\Phi(u) \leq 0$ for all $u \in E_{\mathcal{H}}$ with $\|u\| \geq R_{E_{\mathcal{H}}}$. 
Proof It suffices to show that $\Phi(u) \rightarrow-\infty$ in $E_{\mathcal{H}}$ as $\|u\| \rightarrow \infty$. For any $u \in E_{\mathcal{H}}$, let $u=$ $u_{1}^{+}+u^{-}+u^{0}$, where $u_{1}^{+} \in \mathcal{H}, u^{-} \in E^{-}, u^{0} \in E^{0}$. Since $\operatorname{dim} \mathcal{H}=1$, then

$$
\left|u_{1}^{+}\right|_{2}^{2}=\left\langle u_{1}^{+}, u\right\rangle_{2} \leq\left|u_{1}^{+}\right|_{v^{\prime}}|u|_{\nu} \leq c\left|u_{1}^{+}\right|_{2}|u|_{\nu},
$$

where $\frac{1}{v^{\prime}}+\frac{1}{v}=1$. Thus $\left|u_{1}^{+}\right|_{v} \leq c\left|u_{1}^{+}\right|_{2} \leq c|u|_{v}$, and together with (4), we obtain

$$
\begin{aligned}
\Phi(u) & =\frac{1}{2}\left\|u^{+}\right\|^{2}-\frac{1}{2}\left\|u^{-}\right\|^{2}-\int_{\mathbb{R}} W(t, u(t)) d t \\
& \leq c\left|u_{1}^{+}\right|_{v}^{2}-\frac{1}{2}\left\|u^{-}\right\|^{2}-c\left|u_{1}^{+}+u^{-}+u^{0}\right|_{v}^{v} \\
& \leq c\left|u_{1}^{+}+u^{-}+u^{0}\right|_{v}^{2}-\frac{1}{2}\left\|u^{-}\right\|^{2}-c\left|u_{1}^{+}+u^{-}+u^{0}\right|_{v}^{v}
\end{aligned}
$$

which shows that $\Phi(u) \rightarrow-\infty$ as $\|u\| \rightarrow \infty$.

As a special case we have

Lemma 8 Assume that the assumptions of Theorem 2 are satisfied. Then, letting $e \in \mathcal{H}$ with $\|e\|=1$, there is $r_{1}>\rho>0$ such that $\sup \Phi(\partial M) \leq \kappa$ where $M:=\left\{u=u^{-}+u^{0}+s e\right.$ : $\left.u^{-}+u^{0} \in E^{-} \oplus E^{0}, s \geq 0,\|u\| \leq r_{1}\right\}$ and $\kappa$ is given by Lemma 6 .

\section{The $(C)_{c}$-sequence}

In this section, we discuss the $(C)_{c}$-sequence of $\Phi$.

Lemma 9 Let $\left(L_{3}\right)-\left(L_{4}\right)$ and $\left(W_{1}\right)-\left(W_{4}\right)$ hold. Then any $(C)_{c}$-sequence is bounded.

Proof Let $\left\{u_{j}\right\} \subset E$ be such that

$$
\Phi\left(u_{j}\right) \rightarrow c \text { and }\left(1+\left\|u_{j}\right\|\right) \Phi^{\prime}\left(u_{j}\right) \rightarrow 0 .
$$

Then, for $C_{0}>0$,

$$
C_{0} \geq \Phi\left(u_{j}\right)-\frac{1}{2} \Phi^{\prime}\left(u_{j}\right) u_{j}=\int_{\mathbb{R}} \tilde{W}\left(t, u_{j}\right) d t
$$

Suppose to the contrary that $\left\{u_{j}\right\}$ is unbounded. Setting $y_{j}=u_{j} /\left\|u_{j}\right\|$, then $\left\|y_{j}\right\|=1,\left|y_{j}\right|_{p} \leq$ $c\left\|y_{j}\right\|=c$ for all $p \geq 2$. Passing to subsequence, $y_{j} \rightarrow y$ in $E$, and $y_{j} \rightarrow y$ in $L^{p}$ for $p \geq 1$.

Note that

$$
\begin{aligned}
o(1) & =\Phi^{\prime}\left(u_{j}\right)\left(u_{j}^{+}-u_{j}^{-}\right) \\
& =\left\|u_{j}\right\|^{2}-\int_{\mathbb{R}} W_{u}\left(t, u_{j}\right)\left(u_{j}^{+}-u_{j}^{-}\right) d t \\
& =\left\|u_{j}\right\|^{2}-\left\|u_{j}\right\|^{2} \int_{\mathbb{R}} \frac{W_{u}\left(t, u_{j}\right)\left(y_{j}^{+}-y_{j}^{-}\right)}{\left\|u_{j}\right\|} d t \\
& =\left\|u_{j}\right\|^{2}\left(1-\int_{\mathbb{R}} \frac{W_{u}\left(t, u_{j}\right)\left(y_{j}^{+}-y_{j}^{-}\right)}{\left\|u_{j}\right\|} d t\right) .
\end{aligned}
$$


From (10), we obtain

$$
\int_{\mathbb{R}} \frac{W_{u}\left(t, u_{j}\right)\left(y_{j}^{+}-y_{j}^{-}\right)}{\left\|u_{j}\right\|} d t \rightarrow 1
$$

Set for $s \geq 0$,

$$
h(s):=\inf \left\{\tilde{W}(t, u): t \in \mathbb{R} \text { and } u \in \mathbb{R}^{N} \text { with }|u| \geq s\right\} .
$$

By $\left(W_{1}\right)$ and $\left(W_{3}\right), h(s)>0$ for all $s>0$, and $h(s) \rightarrow \infty$ as $s \rightarrow \infty$.

For $0 \leq l<m$, let

$$
C_{l}^{m}=\inf \left\{\frac{\tilde{W}(t, u)}{|u|^{2}}: t \in \mathbb{R} \text { with } l \leq|u(t)|<m\right\}
$$

and

$$
\Omega_{j}(l, m)=\left\{t \in \mathbb{R}: l \leq\left|u_{j}(t)\right|<m\right\} .
$$

Then by $\left(W_{3}\right)$ one has $C_{l}^{m}>0$ and

$$
\tilde{W}\left(t, u_{j}\right) \geq C_{l}^{m}\left|u_{j}\right|^{2} \quad \text { for all } t \in \Omega_{j}(l, m) \text {. }
$$

It follows from (8) and (12) that

$$
\begin{aligned}
C_{0} & \geq \int_{\Omega_{j}(0, l)} \tilde{W}\left(t, u_{j}\right) d t+\int_{\Omega_{j}(l, m)} \tilde{W}\left(t, u_{j}\right) d t+\int_{\Omega_{j}(m, \infty)} \tilde{W}\left(t, u_{j}\right) d t \\
& \geq \int_{\Omega_{j}(0, l)} \tilde{W}\left(t, u_{j}\right) d t+C_{l}^{m} \int_{\Omega_{j}(l, m)}\left|u_{j}\right|^{2} d t+h(m)\left|\Omega_{j}(m, \infty)\right| .
\end{aligned}
$$

Using (13) we obtain

$$
\left|\Omega_{j}(m, \infty)\right| \leq \frac{C_{0}}{h(m)} \rightarrow 0,
$$

as $m \rightarrow \infty$ uniformly in $j$, and for any fixed $0<l<m$,

$$
\int_{\Omega_{j}(l, m)}\left|y_{j}\right|^{2} d t=\frac{1}{\left\|u_{j}\right\|^{2}} \int_{\Omega_{j}(l, m)}\left|u_{j}\right|^{2} d t \leq \frac{C_{0}}{C_{l}^{m}\left\|u_{j}\right\|^{2}} \rightarrow 0,
$$

as $j \rightarrow \infty$. It follows from (14) that, for any $s \in[2,+\infty)$,

$$
\int_{\Omega_{j}(m, \infty)}\left|y_{j}\right|^{s} d t \leq\left(\int_{\Omega_{j}(m, \infty)}\left|y_{j}\right|^{2 s} d t\right)^{1 / 2} \cdot\left|\Omega_{j}(m, \infty)\right|^{1 / 2} \leq c\left|\Omega_{j}(m, \infty)\right|^{1 / 2} \rightarrow 0
$$

as $m \rightarrow \infty$ uniformly in $j$.

Let $0<\epsilon<\frac{1}{3}$. By $\left(W_{2}\right)$ there is $l_{\epsilon}>0$ such that

$$
\left|W_{u}(t, u)\right|<\frac{\epsilon}{c}|u|
$$


for all $|u| \leq l_{\epsilon}$. Consequently,

$$
\begin{aligned}
\int_{\Omega_{j}\left(0, l_{\epsilon}\right)} \frac{W_{u}\left(t, u_{j}\right)\left(y_{j}^{+}-y_{j}^{-}\right)\left|y_{j}\right|}{\left|u_{j}\right|} d t & \leq \int_{\Omega_{j}\left(0, l_{\epsilon}\right)} \frac{\epsilon}{c}\left|y_{j}^{+}-y_{j}^{-}\right|\left|y_{j}\right| d t \\
& \leq \frac{\epsilon}{c}\left|y_{j}\right|_{2}^{2}<\epsilon
\end{aligned}
$$

for all $j$.

Set $\sigma^{\prime}:=p / 2$. By $\left(W_{4}\right),(16)$ and Hölder inequality, we can take $m_{\epsilon} \geq r$ large enough such that

$$
\begin{aligned}
& \int_{\Omega_{j}\left(m_{\epsilon}, \infty\right)} \frac{W_{u}\left(t, u_{j}\right)\left(y_{j}^{+}-y_{j}^{-}\right)\left|y_{j}\right|}{\left|u_{j}\right|} d t \\
& \quad \leq\left(\int_{\Omega_{j}\left(m_{\epsilon}, \infty\right)} \frac{\left|W_{u}\left(t, u_{j}\right)\right|^{\sigma}}{\left|u_{j}\right|^{\sigma}} d t\right)^{1 / \sigma}\left(\int_{\Omega_{j}\left(m_{\epsilon}, \infty\right)}\left(\left|y_{j}^{+}-y_{j}^{-}\right|\left|y_{j}\right|\right)^{\sigma^{\prime}} d t\right)^{1 / \sigma^{\prime}} \\
& \quad \leq\left(\int_{\Omega_{j}\left(m_{\epsilon}, \infty\right)} c_{1} \tilde{W}\left(t, u_{j}\right) d t\right)^{1 / \sigma}\left(\int_{\mathbb{R}^{N}}\left(\left|y_{j}^{+}-y_{j}^{-}\right|\right)^{p} d t\right)^{1 / p}\left(\int_{\Omega_{j}\left(m_{\epsilon}, \infty\right)}\left|y_{j}\right|^{p} d t\right)^{1 / p} \\
& \quad \leq \epsilon
\end{aligned}
$$

for all $j$. Note that there is $C=C(\epsilon)>0$ independent of $j$ such that $\left|W_{u}\left(t, u_{j}\right)\right| \leq C\left|u_{j}\right|$ for $t \in \Omega_{j}\left(l_{\epsilon}, m_{\epsilon}\right)$. By (15) there is $j_{0}$ such that

$$
\begin{aligned}
\int_{\Omega_{j}\left(l_{\epsilon}, m_{\epsilon}\right)} \frac{W_{u}\left(t, u_{j}\right)\left(y_{j}^{+}-y_{j}^{-}\right)\left|y_{j}\right|}{\left|u_{j}\right|} d t & \leq C \int_{\Omega_{j}\left(l_{\epsilon}, m_{\epsilon}\right)}\left|y_{j}^{+}-y_{j}^{-}\right|\left|y_{j}\right| d t \\
& \leq C\left|y_{j}\right|_{2}\left(\int_{\Omega_{j}\left(l_{\epsilon}, m_{\epsilon}\right)}\left|y_{j}\right|^{2} d t\right)^{1 / 2} \\
& \leq \epsilon
\end{aligned}
$$

for all $j \geq j_{0}$. By (17)-(19), one has

$$
\limsup _{j \rightarrow \infty} \int_{\mathbb{R}} \frac{W_{u}\left(t, u_{j}\right)\left(y_{j}^{+}-y_{j}^{-}\right)}{\left\|u_{j}\right\|} d t \leq 3 \epsilon<1
$$

which contradicts with (10). The proof is complete.

Lemma 10 Under the assumptions of Theorem 2, $\Psi$ is nonnegative, weakly sequentially lower semi-continuous, and $\Psi^{\prime}$ is weakly sequentially continuous. Moreover, $\Psi^{\prime}$ is compact.

Proof We follow the idea of [33]. Clearly, by assumptions, $\Psi(u) \geq 0$. Let $u_{j} \rightarrow u$ in $E$. By Lemma $10, u_{j} \rightarrow u$ in $L^{p}(\mathbb{R})$ for $p \geq 2$, and $u_{j}(t) \rightarrow u(t)$ a.e. $t \in \mathbb{R}$. Hence $W\left(t, u_{j}\right) \rightarrow W(t, u)$ for a.e. $t \in \mathbb{R}$. Thus, it follows from Fatou's lemma that

$$
\Psi(u)=\int_{\mathbb{R}} W(t, u) d t=\int_{\mathbb{R}} \lim _{j \rightarrow \infty} W\left(t, u_{j}\right) d t \leq \liminf _{j \rightarrow \infty} \int_{\mathbb{R}} W\left(t, u_{j}\right) d t=\liminf _{j \rightarrow \infty} \Psi\left(u_{j}\right),
$$

which shows that the function $\Psi$ is weakly sequentially lower semi-continuous. 
Now we show that $\Psi^{\prime}$ is compact. It is clear that, for any $\varphi \in C_{0}^{\infty}(\mathbb{R})$,

$$
\Psi^{\prime}\left(u_{j}\right) \varphi=\int_{\mathbb{R}} W_{u}\left(t, u_{j}\right) \varphi d t \rightarrow \int_{\mathbb{R}} W_{u}(t, u) \varphi d t=\Psi^{\prime}(u) \varphi
$$

Since $C_{0}^{\infty}(\mathbb{R})$ is dense in $E$, for any $v \in E$, we take $\varphi_{n} \in C_{0}^{\infty}(\mathbb{R})$ such that

$$
\left\|\varphi_{n}-v\right\| \rightarrow 0 \quad \text { as } j \rightarrow \infty
$$

By (6), one has

$$
\begin{aligned}
\left|\Psi^{\prime}\left(u_{j}\right) v-\Psi^{\prime}(u) v\right| \leq & \left|\left(\Psi^{\prime}\left(u_{j}\right)-\Psi^{\prime}(u)\right) \varphi_{n}\right|+\left|\left(\Psi^{\prime}\left(u_{j}\right)-\Psi^{\prime}(u)\right)\left(v-\varphi_{n}\right)\right| \\
\leq & \left|\left(\Psi^{\prime}\left(u_{j}\right)-\Psi^{\prime}(u)\right) \varphi_{n}\right| \\
& \quad+c \int_{\mathbb{R}}\left(|u|+\left|u_{j}\right|+|u|^{p-1}+\left|u_{j}\right|^{p-1}\right)\left|v-\varphi_{n}\right| \\
\leq & \left|\left(\Psi^{\prime}\left(u_{j}\right)-\Psi^{\prime}(u)\right) \varphi_{n}\right|+c\left\|v-\varphi_{n}\right\| .
\end{aligned}
$$

For any $\epsilon>0$, fix $n$ so that $\left\|\nu-\varphi_{n}\right\|<\epsilon / 2 c$. By (21) there exists $j_{0}$ such that

$$
\left|\left(\Psi^{\prime}\left(u_{j}\right)-\Psi^{\prime}(u)\right) \varphi_{n}\right|<\epsilon / 2 \text { for all } j \geq j_{0} .
$$

Then $\left|\left(\Psi^{\prime}\left(u_{j}\right)-\Psi^{\prime}(u)\right) \varphi_{n}\right|<\epsilon$ for all $j \geq j_{0}$, which proves the weakly sequentially continuity. Therefore, $\Psi^{\prime}$ is compact by the weakly continuity of $\Psi^{\prime}$ since $E$ is a Hilbert space.

Lemma 10 implies that $\Phi^{\prime}$ is weakly sequentially continuous, i.e., if $u_{j} \rightarrow u$ in $E$, then $\Phi^{\prime}\left(u_{j}\right) \rightarrow \Phi^{\prime}(u)$. Let $\left\{u_{j}\right\}$ be an arbitrary $(C)_{c}$-sequence, by Lemma 9 , it is bounded, up to a subsequence, we may assume $u_{j} \rightarrow u$ in $E$. Plainly, $u$ is a critical point of $\Phi$.

Lemma 11 Under the assumptions of Lemma 9, $\Phi$ satisfies $(C)_{c}$-condition.

Proof Let $\left\{u_{j}\right\}$ be any $(C)_{c}$-sequence. By Lemmas 4, 9, and 10, one has

$$
\begin{aligned}
& \left|\int_{\mathbb{R}}\left(W_{u}\left(t, u_{j}\right)-W_{u}(t, u)\right)\left(u_{j}^{+}-u^{+}\right) d t\right| \\
& \quad \leq\left(\int_{\mathbb{R}}\left|W_{u}\left(t, u_{j}\right)-W_{u}(t, u)\right|^{2} d t\right)^{\frac{1}{2}}\left(\int_{\mathbb{R}}\left|u_{j}^{+}-u^{+}\right|^{2} d t\right)^{\frac{1}{2}} \\
& \quad \leq c\left(\int_{\mathbb{R}}\left|u_{j}^{+}-u^{+}\right|^{2} d t\right)^{\frac{1}{2}} \rightarrow 0
\end{aligned}
$$

and

$$
\begin{aligned}
o(1) & =\left(\Phi^{\prime}\left(u_{j}\right)-\Phi^{\prime}(u), u_{j}^{+}-u^{+}\right) \\
& =\left\|u_{j}^{+}-u^{+}\right\|^{2}+\int_{\mathbb{R}}\left(W_{u}\left(t, u_{j}\right)-W_{u}(t, u)\right)\left(u_{j}^{+}-u^{+}\right) d t \\
& =\left\|u_{j}^{+}-u^{+}\right\|^{2}+o(1) .
\end{aligned}
$$


So $u_{j}^{+} \rightarrow u^{+}$as $j \rightarrow \infty$. Since $\operatorname{dim}\left(E^{-} \oplus E^{0}\right)<\infty$, we have $u_{j}^{-}+u_{j}^{0} \rightarrow u^{-}+u^{0}$, and therefore $u_{j} \rightarrow u$ as $j \rightarrow \infty$ in $E$.

\section{Proof of the theorem}

Proof of Theorem 2 Lemma 8 shows that $\Phi$ possesses the linking structure of Theorem 5, and Lemma 11 implies that $\Phi$ satisfies the $(C)_{c}$-condition. Therefore, by Theorem $5 \Phi$ has at least one critical point $u$.

\section{Competing interests}

The authors declare that they have no competing interests.

\section{Authors' contributions}

Each of the authors, JS, JN and MO contributed to each part of this study equally and read and approved the final version of the manuscript.

\section{Author details}

${ }^{1}$ School of Science, Shandong University of Technology, Zibo, Shandong 255049, China. ${ }^{2}$ Departamento de Análisis Matemático, Facultad de Matemáticas, Universidad de Santiago de Compostela, Santiago de Compostela, 15782, Spain. ${ }^{3}$ Department of Mathematics, Faculty of Science, King Abdulaziz University, P.O. Box 80203, Jeddah, 21589, Saudi Arabia.

\section{Acknowledgement}

The research of J. J. Nieto was partially supported by the Ministerio de Economía y Competitividad and FEDER, project MTM2010-15314. The research of J. Sun was supported by the National Natural Science Foundation of China (Grant No. 11201270, 11271372), Shandong Natural Science Foundation (Grant No. ZR2012AQ010).

Received: 12 April 2012 Accepted: 8 June 2012 Published: 5 July 2012

\section{References}

1. Coti-Zelati, V, Rabinowitz, PH: Homoclinic orbits for second order Hamiltonian systems possessing superquadratic potentials. J. Am. Math. Soc. 4, 693-727 (1991)

2. Mawhin, J, Willem, M: Critical Point Theory and Hamiltonian Systems. Applied Mathematical Sciences, vol. 74. Springer, New York (1989)

3. Rabinowitz, PH: Homoclinic orbits for a class of Hamiltonian systems. Proc. R. Soc. Edinb. A 114, 33-38 (1990)

4. Ambrosetti, A, Coti-Zelati, V: Multiple homoclinic orbits for a class of conservative systems. Rend. Semin. Mat. Univ. Padova 89, 177-194 (1993)

5. Chen, G, Ma, S: Periodic solutions for Hamiltonian systems without Ambrosetti-Rabinowitz condition and spectrum. J. Math. Anal. Appl. 379, 842-851 (2011)

6. Ding, J, Xu, J, Zhang, F: Existence of homoclinic orbits for Hamiltonian systems with superquadratic potentials. Abstr. Appl. Anal. 2009, Art. ID 128624 (2009)

7. Ding, Y: Existence and multiplicity results for homoclinic solutions to a class of Hamiltonian systems. Nonlinear Anal. 25, 1095-1113 (1995)

8. Izydorek, M, Janczewska, J: Homoclinic solutions for a class of second order Hamiltonian systems. J. Differ. Equ. 219, 375-389 (2005)

9. Kim, Y: Existence of periodic solutions for planar Hamiltonian systems at resonance. J. Korean Math. Soc. 48, 1143-1152 (2011)

10. Omana, W, Willem, M: Homoclinic orbits for a class of Hamiltonian systems. Differ. Integral Equ. 5, 1115-1120 (1992)

11. Paturel, E: Multiple homoclinic orbits for a class of Hamiltonian sytems. Calc. Var. Partial Differ. Equ. 12, 117-143 (2001)

12. Rabinowitz, PH: Variational methods for Hamiltonian systems. In: Handbook of Dynamical Systems, vol. 1, Part 1, Chapter 14, pp. 1091-1127. North-Holland, Amsterdam (2002)

13. Rabinowitz, PH, Tanaka, K: Some results on connecting orbits for a class of Hamiltonian systems. Math. Z. 206 473-499 (1991)

14. Séré, E: Existence of infinitely many homoclinic orbits in Hamiltonian systems. Math. Z. 209, 133-160 (1992)

15. Sun, J, Chen, H, Nieto, JJ: Homoclinic solutions for a class of subquadratic second-order Hamiltonian systems. J. Math. Anal. Appl. 373, 20-29 (2011)

16. Sun, J, Chen, H, Nieto, JJ: Homoclinic orbits for a class of first-order nonperiodic asymptotically quadratic Hamiltonian systems with spectrum point zero. J. Math. Anal. Appl. 378, 117-127 (2011)

17. Sun, J, Chen, H, Nieto, JJ: On ground state solutions for some non-autonomous Schrödinger systems. J. Differ. Equ. $252,3365-3380(2012)$

18. Tang, X, Xiao, L: Homoclinic solutions for non-autonomous second-order Hamiltonian systems with a coercive potential. J. Math. Anal. Appl. 351, 586-594 (2009)

19. Tang, X, Lin, X: Homoclinic solutions for a class of second order Hamiltonian systems. J. Math. Anal. Appl. 354, 539-549 (2009)

20. Wan, L, Tang, C: Existence and multiplicity of homoclinic orbits for second order Hamiltonian systems without (AR) condition. Discrete Contin. Dyn. Syst., Ser. B 15, 255-271 (2011) 
21. Zhang, P, Tang, C: Infinitely many periodic solutions for nonautonomous sublinear second-order Hamiltonian systems. Abstr. Appl. Anal. 2010, Art. ID 620438 (2010)

22. Zhang, Q, Liu, C: Infinitely many homoclinic solutions for second order Hamiltonian systems. Nonlinear Anal. 72 894-903 (2010)

23. Nieto, JJ: Variational formulation of a damped Dirichlet impulsive problem. Appl. Math. Lett. 23, 940-942 (2010)

24. Sun, J, Chen, H, Nieto, JJ: Infinitely many solutions for second-order Hamiltonian system with impulsive effects. Math. Comput. Model. 54, 544-555 (2011)

25. Sun, J, Chen, H, Nieto, JJ, Otero-Novoa, M: The multiplicity of solutions for perturbed second-order Hamiltonian systems with impulsive effects. Nonlinear Anal. 72, 4575-4586 (2010)

26. Xiao, J, Nieto, JJ: Variational approach to some damped Dirichlet nonlinear impulsive differential equations. J. Franklin Inst. 348, 369-377 (2011)

27. Wu, X Zhang, W: Existence and multiplicity of homoclinic solutions for a class of damped vibration problems. Nonlinear Anal. 74, 4392-4398 (2011)

28. Zhang, Z, Yuan, R: Homoclinic solutions for some second-order non-autonomous systems. Nonlinear Anal. 71, 5790-5798 (2009)

29. Zhu, W: Existence of homoclinic solutions for second order systems. Nonlinear Anal. 75, 2455-2463 (2012)

30. Willem, M, Zou, W: On a Schrödinger equation with periodic potential and spectrum point zero. Indiana Univ. Math. J. 52, 109-132 (2003)

31. Wang, J, Xu, J, Zhang, F: Homoclinic orbits for a class of Hamiltonian systems with superquadratic or asymptotically quadratic potentials. Commun. Pure Appl. Anal. 10, 269-286 (2011)

32. Ding, Y, Szulkin, A: Bound states for semilinear Schrödinger equations with sign changing potential. Calc. Var. Partial Differ. Equ. 29, 397-419 (2007)

33. Ding, Y: Multiple homoclinics in a Hamiltonian system with asymptotically or super linear terms. Commun. Contemp. Math. 8, 453-480 (2006)

doi:10.1186/1687-1847-2012-102

Cite this article as: Sun et al.: On homoclinic orbits for a class of damped vibration systems. Advances in Difference Equations 2012 2012:102.

\section{Submit your manuscript to a SpringerOpen ${ }^{\circ}$ journal and benefit from:}

- Convenient online submission

- Rigorous peer review

- Immediate publication on acceptance

- Open access: articles freely available online

- High visibility within the field

- Retaining the copyright to your article 